\title{
Variation in fatty acid composition of milk and cheese from cows grazed on two alpine pastures
}

\author{
Luca FAlChero $^{1 *}$, Giampiero Lombardi ${ }^{1}$, Alessandra Gorlier ${ }^{1}$, Michele Lonati ${ }^{1}$, \\ Miriam OdoARdi $^{2}$, Andrea CAVAllero ${ }^{1}$ \\ ${ }^{1}$ University of Turin, Department AGROSELVITER, Turin, Italy \\ ${ }^{2}$ CRA-FLC, Lodi, Italy
}

Received 12 November 2009 - Revised 4 May 2010 - Accepted 4 May 2010

Published online 2 August 2010

\begin{abstract}
The aim of this study was to verify the variation in fatty acid composition of milk, and derived cheese, when dairy cows were grazed on different alpine vegetation types. An experimental design was developed to highlight the effect of pasture composition on milk and cheese fatty acid profiles, thereby mitigating the other variables (altitude, animal physiology, and animal management). Two dairy cow groups were grazed on two different key alpine vegetation types (Type 1: Trifolium alpinum, Nardus stricta, and Carex sempervirens and Type 2: Festuca nigrescens, Alchemilla xanthochlora, and Phleum alpinum). Bulk milk was collected separately from each group for 6 days, from which 12 artisanal cheeses were produced and ripened for 60 days. The fatty acid (FA) composition of the bulk milks and cheeses varied with the type of vegetation. Milk and cheese derived from cows that were grazed on Trifolium alpinum-dominated pastures were richer in long-chain FAs, unsaturated and monounsaturated FAs (particularly oleic acid), and odd-chain saturated FAs (such as C15:0 pentadecanoic and C17:0 heptadecanoic acids) while those from Festuca nigrescens pastures contained more short- and medium-chain FAs, saturated FAs, and $\alpha$-linolenic acid. Furthermore, our results showed that in alpine grazing systems, milk and cheese FA profiles changed when cows were grazed on pastures with a different botanical composition, probably due to differences in forage quality and concentration of bioactive secondary metabolites. These results have to be taken into account for the valorization of the nutritional characteristics and for the traceability of grass-fed dairy products.
\end{abstract}

fatty acid / milk / cheese / grassland biodiversity / alpine pasture / grazing

摘要 - 两种高山放牧奶牛的乳脂肪酸和干酪脂肪酸组成的变化。本文证实了不同类型高山 牧场放牧的奶牛乳脂肪酸和干酪脂肪酸组成的变化。着重研究了牧草构成对乳及其干酪脂 肪酸的影响、目的是降低其他因素的影响（如：海拔，奶牛的生理特性以及奶牛的管理状 况）。将奶牛分为两组、分别在两种不同类型的高山牧场上放牧（牧场种类 1: 主要以三叶 高山亚麻草、那杜草和苔属地中海柏木草为主; 牧场种类 2: 主要以纬状羊茅草、黄裙羽衣 草和高山梯牧草为主）。分别从两组奶牛中采集大量的牛奶、时间持续 $6 \mathrm{~d}$ 、同时也分别从 两组手工生产的干酪中采集了 12 个样本、干酪成熟期为 60 天。实验结果表明：乳和干酪 的脂肪酸组成是随高山牧草构成而变化的。以三叶高山亚麻草牧场为主（种类 1) 的奶牛 的奶脂中含有较高的长链脂肪酸、不饱和脂肪酸、单不饱和脂肪酸 (特别是油酸含量较高) 以及奇数链脂肪酸 (如十五烷酸和十七烷酸)。而那些以纬状羊茅草牧场为主 (种类 2) 的 奶牛的乳脂中含有较高的短链、中链脂肪酸、饱和脂肪酸和 $\alpha$-亚麻酸。此外、结果显示在 高山牧草体系中、当牧草有不同的植被构成时、乳和干酪的脂肪酸组成都会发生变化、原

*Corresponding author (通讯作者): luca.falchero@unito.it 
因可能是由于牧草的质量和生物次级代谢产物不同所导致的。本实验结果可以作为稳定乳 的营养特性和牧草喂养乳制品可追溯性的参考值。

\title{
脂肪酸 / 乳 / 干酪 / 草原生物多样性 / 高山放牧 / 牧草
}

\begin{abstract}
Résumé - Variation de la composition en acides gras dans le fromage et le lait provenant de vaches ayant brouté deux types de pâtures alpines. Cette étude a eu pour but de vérifier la variation de la composition en acides gras du lait et du fromage dérivé, alors que les vaches broutaient différents types de végétation alpine. Le plan d'expérience a été développé pour souligner l'effet de la composition de la pâture sur le profil en acides gras du lait et du fromage, en atténuant les autres variables (altitude, physiologie de l'animal et conduite du troupeau). Deux groupes de vaches laitières ont brouté deux types de végétation alpine (type 1: Trifolium alpinum, Nardus stricta et Carex sempervirens, et type 2 : Festuca nigrescens, Alchemilla xanthochlora et Phleum alpinum). Le lait de mélange a été collecté séparément dans chaque groupe pendant 6 jours, puis 12 fromages artisanaux ont été fabriqués et affinés pendant 60 jours à partir de ces laits. La composition en acides gras (AG) des laits de mélange et des fromages variait avec le type de végétation. Le lait et les fromages provenant des vaches ayant brouté les pâtures à dominante Trifolium alpinum étaient plus riches en AG à longue chaîne, insaturés et mono-insaturés (acide oléique en particulier), et en AG saturés à nombre impair de carbone (tels que l'acide pentadécanoïque C15:0 et l'acide heptadécanoïque C17:0), tandis que le lait et les fromages provenant des vaches ayant brouté les pâtures à dominante Festuca nigrescens contenaient plus d'AG à courte chaîne ou à chaîne moyenne, d'AG saturés et d'acide alpha-linolénique. En outre, les résultats montraient que dans les systèmes de pâture alpine, les profils en AG du lait et des fromages changeaient lorsque les vaches broutaient des pâtures de composition botanique différente, probablement en raison des différences de qualité de fourrage et de concentration en métabolites secondaires bioactifs. Ces résultats doivent être pris en considération dans la valorisation des caractéristiques nutritionnelles et la traçabilité des produits laitiers provenant d'une alimentation à l'herbe.
\end{abstract}

acide gras / lait / fromage / biodiversité des pâtures / pâture alpine / pâturage

\section{INTRODUCTION}

The fatty acid (FA) composition of milk and dairy products has been widely investigated to obtain a greater understanding of their effect on human health. It has been established that many saturated $(\mathrm{C} 12: 0$, $\mathrm{C} 14: 0$, and $\mathrm{C} 16: 0)$ and trans fatty acids increase the risk of coronary heart disease [42]. On the contrary, other unsaturated fatty acids have been shown to act mostly as essential compounds for mammals by protecting against cardiovascular diseases and inhibiting degenerative cell proliferation [49].

Beyond the human health effects, FA profiling is a useful tool, when used singly or in combination with other methods, for milk and cheese quality and tracing their origin [17], and to verify product authenticity.
Fatty acid composition and concentration in milk depend on two main factors: (i) animal physiology/characteristics including breed, age, lactation stage, and response to environmental factors [36, 40]; and (ii) animal diet $[7,8]$, with particular emphasis on the type and level of supplementation, as well as forage type (hay, silage, and fresh herbage) and amount [16]. A diet supplied with fresh forage may produce milk richer in FAs with nutraceutical properties, such as polyunsaturated fatty acids (PUFA), in particular conjugated linoleic acid (CLA) and n-3 FA [12, 20]. In fact the main dietary precursor of CLA and $n-3$ is $\alpha$-linolenic acid (ALA) which accounts for about $50 \%-75 \%$ of total FA content in fresh herbage, depending on forage botanical composition [15] and phenological stage [19]. 
Grass-fed cow's milk exhibits important FA differences when it is derived from highland pastures as opposed to lowland pastures [9]. However, the exact origin of such differences remains uncertain due to the interplay of several factors:

(i) grazing energy requirement variations can affect body fat distribution [6],

(ii) altitude variations can affect oxygen metabolism [9] and can cause an energy deficit [33],

(iii) grazing land composition that differs in fibre content and/or in plant secondary metabolite (PSM) concentrations (terpenes, polyphenols, and PUFAs) which can inhibit FA ruminal bio-hydrogenation [34, 37, 44], and/ or modify FA duodenal flow [49], and

(iv) FA profiles of various forages can result in differing intakes of individual FA [15].

The botanical composition of grasslands can be remarkably different not only between mountain and lowland pastures, but also among alpine grasslands. Their effect on FA composition has not been investigated in the Alps until now, even though cheese makers and scientists empirically realize that the exploitation of different highland pastures has resulted in milk and dairy product variations.

We compared the FA composition of cow-produced milk and cheese from two different highland pastures important to summer cheese production. One pasture was largely dominated by Trifolium alpinum, from a genus rich in polyphenolic compounds [39]. The other pasture was rich both in high-forage quality species (Festuca nigrescens, Phleum alpinum, and Trifolium repens) and in other species, such as Alchemilla xanthochlora and Polygonum bistorta, belonging to families with high PSM content [18, 31, 38].

\section{MATERIALS AND METHODS}

\subsection{Experiment organization}

The experiment was performed at Alpe Valcavera (Cuneo; latitude 44 $22^{\prime} 54^{\prime \prime}$ and longitude $\left.7^{\circ} 6^{\prime} 43^{\prime \prime}\right)$, located in the southwest Alps, during the summer of 2007 (11th July-4th August). Festuca nigrescens (F) and Trifolium alpinum $(\mathrm{T})$ vegetation areas at altitudes between 2230 and $2440 \mathrm{~m}$ a.s.l. were mapped before exploitation. Soils in the area overlay a limestone bedrock and shared similar climatic (mean annual rainfall: $1040 \mathrm{~mm}$; mean annual temperature: $2.6^{\circ} \mathrm{C}$ ) and topographic conditions (mean slope: $3-5^{\circ}$; main exposition: $90^{\circ}$ ). Vegetation composition was surveyed using a vertical point-quadrant method [10] along $25 \mathrm{~m}$ permanent transects.

Treatment areas $\mathrm{F}$ and $\mathrm{T}$ were selected and then fenced to create eight paddocks (average surface area: $1.1 \mathrm{ha}$ ) for exploitation by two groups (G1 and G2) of nine dairy cows, each composed of three Italian Red-Pie, one Alpine Grey, one Tarantaise, and four crossbreeds. All were multiparous, in late lactation (216 $\pm 43 \mathrm{~d})$, and adapted to alpine grazing environments. The groups were balanced for daily milk productions $\left(16.5 \pm 5.0 \mathrm{~kg} \cdot \mathrm{d}^{-1} \cdot \mathrm{cow}^{-1}\right)$.

During Period 1, G1 and G2 exploited two $\mathrm{F}$ and two $\mathrm{T}$ paddocks, respectively. Prior to the experiment, each group had grazed a paddock for 6 days (A1, d 1-6) to allow the rumen to fully adapt to the vegetation. Then, each group moved to experimental paddocks where cows grazed for another 6 days (P1, d 7-12). At the end of Period 1, following a cross-over design, the two groups were crossed (G1 to $\mathrm{T}$ paddocks and $\mathrm{G} 2$ to $\mathrm{F}$ paddocks) and two pairs of new paddocks were exploited according to the same scheme (A2, d 13-18 and P2, d 19-24).

The cows grazed for full days and received no supplementation during exploitation except mineral integration. 


\subsection{Vegetation sampling and analyses}

\subsubsection{Sampling}

Bulk vegetation samples (100 g each in six replicates for FA analysis and $500 \mathrm{~g}$ each in three replicates for chemical composition) were collected from experimental $\mathrm{F}$ and $T$ paddocks the day before experimental periods started (d 6 and 18 before periods $\mathrm{P} 1$ and P2, respectively). Samples were weighed, placed in plastic bags, and immediately refrigerated. In the laboratory, samples for FA analysis were stored at $-18{ }^{\circ} \mathrm{C}$, while samples for chemical composition analysis were oven-dried as described below.

\subsubsection{FA analysis}

The forage fat fraction (expressed as $\mathrm{g} \cdot \mathrm{kg}^{-1}$ of DM content) was extracted for FA composition analysis with petroleum ether (Soxtec System HT.6 Extraction Unit and Service Unit; Foss Tecator, Hoganas, Sweden) according to the methodology reported in [47]. After extraction, the forage fat fraction was analysed by gas-chromatography (GC) as described below for milk.

\subsubsection{Chemical composition}

Samples were dried in a forced draft oven to a constant weight at $60{ }^{\circ} \mathrm{C}$ to determine forage dry matter (DM) content. After grinding in a Cyclotec mill (Foss Tecator, Hoganas, Sweden) samples were sieved through a $1 \mathrm{~mm}$ screen and analysed for crude protein (CP) and ash [2], neutral detergent fibre (NDF), acid detergent fibre (ADF), and acid detergent lignin (ADL) [1], organic matter digestibility (OMD) [27], and forage energetic value as milk forage unit (UFL) [5]. Duplicate analyses for all parameters were performed. Duplicate values were subsequently averaged and means were considered as observations for statistical analyses.

\subsection{Assessment of FA intake and botanical composition of ingested forage}

Forage allowance and daily DM intake by the cows were estimated by the sward cutting method [45]; a measured proportion (12 $\times$ $0.41 \mathrm{~m}^{2}$ for each paddock) of the area allotted to the animals was harvested to calculate the total herbage offered to animals. The amount of herbage at the end of each grazing event was measured using the same methodology. The difference between pre- and postevent measurements, adjusted to account for re-growth $\left(5 \mathrm{~kg} \mathrm{DM} \cdot \mathrm{ha}^{-1} \cdot \mathrm{d}^{-1}\right.$ for $\mathrm{T}$, and $10 \mathrm{~kg} \mathrm{DM} \cdot \mathrm{ha}^{-1} \cdot \mathrm{d}^{-1}$ for $\mathrm{F}$, as reported in [4]), allowed the phytomass consumed directly within the paddocks to be assessed. Mean values of DM intake per treatment were calculated, then daily cow FA ingestion calculations were performed using estimated total DM intake divided by grazing event length.

The botanical composition of ingested forage was assessed on the same transects used for botanical surveys by the defoliation index (DI) method [28] at the end of the exploitation period in each experimental paddock (d 12 and 24), with the contribution to defoliation index (CDI) being reported for each species. Using this method, the consumption of each pasture species was rated by an index ranging from $0 \%$ to $100 \%$, estimated for each plant occurring in the transect three levels of defoliation (ungrazed plants $=0$, partially grazed plants $=0.5$, and intensively grazed plants $=1$ ). The DI of species $j$ was calculated as:

$$
\begin{aligned}
\mathrm{DI}_{j}= & (a \times 0+b \times 0.5+c \times 1) / \\
& (a+b+c) \times 100
\end{aligned}
$$

where $a, b$, and $c$ are the frequencies of ungrazed, partially grazed, intensively grazed plants along the transect, respectively, while the sum $a+b+c$ is the overall 
species frequency $\left(\mathrm{FS}_{j}\right)$. Then, CDI of species $j$ was calculated as:

$$
\mathrm{CDI}_{j}=\left(\mathrm{DI}_{j} \times \mathrm{SC}_{j}\right) / \Sigma\left(\mathrm{DI}_{j} \times \mathrm{SC}_{j}\right),
$$

where $\mathrm{SC}_{j}$ is the specific contribution to vegetation composition, computed as the ratio between $\mathrm{FS}_{j}$ and the sum of the frequencies of all the species noted along the transect. CDI expressed single species relative exploitation in relation to the total exploitation.

\subsection{Milk sampling and analyses}

\subsubsection{Sampling}

Cows were milked twice a day at 7:00 (morning milk) and 18:00 (evening milk) in the paddocks using the same milking machine and milk yields were recorded. Three "bulk" tank-milk samples (evening milk + morning milk; $100 \mathrm{~mL}$ ) per day were collected for each treatment on days 9, 10, 11 and 21, 22, 23. Samples were placed into plastic vessels and immediately refrigerated before transportation to the laboratory, where they were stored at $-18{ }^{\circ} \mathrm{C}$.

\subsubsection{FA analysis}

The extraction and quantification of FAs from milk was performed using the following methods ISO 5508:1990 [25] and EN ISO 5509:2000 [26]. Milk samples were defrosted and, after the addition of ammonia, mixed with $4 \mathrm{~mL}$ of isooctane. Glycerides were transesterified to their corresponding FA methyl esters with a solution of potassium hydroxide in methanol $\left(2 \mathrm{~mol} \cdot \mathrm{L}^{-1}\right)$. After centrifugation (3000 rpm per $10 \mathrm{~min}$ ), FAs were separated by GC with an SP-2330 fused-silica capillary column $(60 \mathrm{~m} \times 0.25 \mathrm{~mm}, 0.2 \mu \mathrm{m}$; Supelco, Bellefonte, PA, USA) on an Agilent 7890A (Agilent, Santa Clara, CA, USA) gas chromatograph equipped with autosampler and with flame ionization detector. The oven temperature was set to
$70{ }^{\circ} \mathrm{C}$ and then increased to $170{ }^{\circ} \mathrm{C}$ in $15^{\circ} \mathrm{C} \cdot \mathrm{min}^{-1}$ increments. Thereafter, the temperature was increased to $220^{\circ} \mathrm{C}$ at $3{ }^{\circ} \mathrm{C} \cdot \mathrm{min}^{-1}$ and held for $15 \mathrm{~min}$. Inlet and detector temperatures were $250{ }^{\circ} \mathrm{C}$. Hydrogen was used as carrier gas.

Quantification was made using reference standards (Supelco 37 component FAME mix; Larodan $\mathrm{AB} c 9 t 11-\mathrm{CLA})$ and the results expressed in absolute values of $g$ FAME per $100 \mathrm{~g}$ fat. Replicate analysis was averaged, which yielded 12 values for each milkrelated variable for statistical purposes.

\subsection{Cheese sampling and analyses}

\subsubsection{Sampling}

Raw whole milk was converted into Nostrale d'Alpe, a typical regional cheese, during the morning of days 9, 10, 11 and $21,22,23$. Six cheeses were obtained from the distinct curds of each treatment $(\mathrm{F}$ and $\mathrm{T})$. After ripening $(60 \mathrm{~d})$, three samples were collected (100-150 g) from each wheel, placed in plastic bags, and vacuum-stored at $-18{ }^{\circ} \mathrm{C}$ until analysis.

\subsubsection{FA analysis}

The cheese samples were defrosted and submitted to cold chloroform extraction for $2 \mathrm{~h}$ (1:2). After chloroform cold evaporation by Rotovapor, the fat extract was treated using the same methodology used for milk samples. As with milk, the daily analytical replicates were averaged, producing 12 values per cheese-related variable for statistical purposes.

\subsection{Statistics}

A general linear ANOVA model $\left(Y_{i j}=\right.$ $\left.\mu+P_{i=1,2}+Q_{j=1,2}+\xi_{i j}\right)$ was used to evaluate the effect of vegetation on milk and cheese FA composition in which $\mu$ represented the overall mean and $\xi_{i j}$ the residual error. $P_{i}=1,2$ represented the two levels 
of vegetation treatment ( $\mathrm{F}$ and $\mathrm{T})$ introduced as fixed factors in the model, and $Q_{j}=1,2$ represented the two experimental periods (P1 and P2), expressing time-line variations such as progression of animal lactation and changes in plant phenology as random factors. The 6-day adaptation time between the first and second trial periods allowed us to treat the carryover effect from the first to second treatment as null [29], but prevented assessment of $P \times Q$ interaction [13].

All analyses were performed using the SPSS version 16.0 statistical package (SPSS, 2007).

\section{RESULTS}

\subsection{Vegetation composition, forage production, and quality}

Table I reports the vegetation composition of paddocks $\mathrm{F}$ and $\mathrm{T}$ and their associated PSM richness values. Paddock F vegetation had a higher specific contribution of PSM-rich species than T $(42-46 \%$ vs. $29-27 \%$ ) because of the abundance of species from the Compositae, Rosaceae, Polygonaceae, and Fabaceae families, all generally rich in either volatiles [38] or polyphenols [23, 31]. Paddock T vegetation was dominated by Trifolium alpinum, a species from the Fabaceae family known to be rich in polyphenols [39].

$\mathrm{F}$ pasture biomass production was almost double that of T (Tab. II). The forage had a high DM content during the entire experiment, particularly in $\mathrm{T}$. We also found that the content of $\mathrm{CP}$, which decreased during the season for both types, remained significantly higher whenever Fabaceae species were more abundant.

Neither OMD nor energy values differed significantly across vegetation types or periods. However, we did observe a lower NDF content in $\mathrm{F}$ and found ADL content differed by period.

\subsection{Fatty acid intake}

Cow daily DM intake did not statistically differ between treatments (Tab. III), even if a tendency $(P=0.19)$ for higher values in $\mathrm{F}$ were noted $\left(16.5\right.$ vs. $\left.11.7 \mathrm{~kg} \cdot \mathrm{LU}^{-1} \cdot \mathrm{d}^{-1}\right)$.

With regard to forage FA composition, only odd-chain saturated fatty acids (0.60.4 vs. $14.9-12.5 \mathrm{~g} \cdot 100 \mathrm{~g}^{-1} \mathrm{DM}$ for C15:0, 0.5-0.4 vs. 5.0-4.2 g.100 g ${ }^{-1} \mathrm{DM}$ for $\mathrm{C} 17: 0)$ and palmitic acid (C16:0; 34.626.2 vs. $\left.71.6-60.4 \mathrm{~g} \cdot 100 \mathrm{~g}^{-1} \mathrm{DM}\right)$ showed lower values $(P<0.05)$ in $\mathrm{F}$ forage. No statistical differences were recorded for pasture type or for the period.

Specific CDI values reported in Table I supported the evidence that grazing cows selected species almost proportionately to their abundance in the sward, particularly for those species having a high SC. The highest specific CDI values appeared to be related to the particular palatability properties (as for Polygonum bistorta). Only a few low-growing species (Alchemilla xanthochlora or Trifolium repens) showed low CDI values. The ingestion of species rich in PSM was confirmed to be higher for cows grazing $\mathrm{F}$ paddocks than $\mathrm{T}$, as expressed by CDI (25-40\% vs. $12-18 \%)$. These results allowed us to consider the mean FA content of "bulk" vegetation samples to be representative of FA content of ingested forage.

As a result, cows ingested more of the following acids when grazed on $\mathrm{F}$ versus $\mathrm{T}$ pastures: lauric $(\mathrm{C} 12: 0 ; P<0.05)$, myristic (C14:0; $P<0.05)$, oleic ( $99-\mathrm{C} 18: 1 ; P<$ 0.05 ), and $\gamma$-linolenic (GLA - C18:3 n-6; $P<0.01)$. We could not statistically confirm the higher intake of linoleic acid (LA - C18:2 $\mathrm{n}-6 ; P=0.14)$ in F. Daily intake of palmitic $(\mathrm{C} 16: 0 ; P<0.05)$ and linear odd-chain saturated fatty acids (C15:0 and C17:0; $P<0.01)$ was lower in treatment $\mathrm{F}$. We observed no intake differences between treatments for either stearic (C18:0) or $\alpha$-linolenic acid (ALA - C18:3 n-3). 
Table I. Composition of Festuca nigrescens (F) and Trifolium alpinum (T) pastures: mean specific contribution (SC, \%), mean contribution to defoliation index (CDI, \%) during experimental period (P1 and P2). Plant secondary metabolites (PSM) richness from bibliographic data in ordinal scale: - poor to +++ very rich. Letters identify statistical differences of SC and CDI within the same vegetation type depending on the experimental paddock.

\begin{tabular}{|c|c|c|c|c|c|c|}
\hline \multirow[t]{2}{*}{ Species } & \multicolumn{2}{|c|}{$\mathrm{SC}$} & \multicolumn{2}{|c|}{ CDI } & \multirow[t]{2}{*}{ PSM } & \multirow[t]{2}{*}{ References } \\
\hline & P1 & P2 & P1 & $\mathrm{P} 2$ & & \\
\hline \multicolumn{7}{|l|}{$\mathrm{F}$} \\
\hline Festuca nigrescens Lam. non Gaudin & $15.95^{\mathrm{a}}$ & $22.20^{\mathrm{b}}$ & 9.90 & 10.11 & - & {$[22,37]$} \\
\hline Alchemilla xanthochlora Rothm. & 13.20 & 13.40 & 3.42 & 3.40 & ++ & {$[17,30]$} \\
\hline Phleum alpinum $\mathrm{L}$. & $4.60^{\mathrm{a}}$ & $11.47^{\mathrm{b}}$ & $5.63^{\mathrm{a}}$ & $9.86^{\mathrm{b}}$ & - & [37] \\
\hline Trifolium repens $\mathrm{L}$. & 8.57 & 7.20 & 4.06 & 2.66 & + & {$[37,38,45]$} \\
\hline Polygonum bistorta $\mathrm{L}$. & 8.01 & 6.01 & 16.19 & 10.27 & +++ & {$[30,37]$} \\
\hline Ranunculus gr. montanus & 4.52 & 6.50 & 1.31 & 1.86 & + & [37] \\
\hline Poa pratensis $\mathrm{L}$. & $8.74^{\mathrm{b}}$ & $0.56^{\mathrm{a}}$ & 5.76 & - & - & {$[37]$} \\
\hline Geum montanum L. & 1.95 & 4.70 & 0.80 & 2.72 & ++ & {$[37]$} \\
\hline Poa alpina $\mathrm{L}$. & 3.42 & 1.96 & 4.07 & 0.00 & - & {$[37]$} \\
\hline Poa annua L. & $4.16^{\mathrm{b}}$ & $0.27^{\mathrm{a}}$ & 2.15 & - & - & [37] \\
\hline Potentilla tabernaemontani Asch. & 1.51 & 2.84 & 0.00 & 1.46 & ++ & {$[37]$} \\
\hline Achillea gr. millefolium & 1.84 & 2.37 & 0.00 & 5.90 & +++ & {$[22,30,37]$} \\
\hline $\begin{array}{l}\text { Trifolium pratense L. ssp. nivale } \\
\text { (Sieber) Asch. et Gr. }\end{array}$ & 2.02 & 1.81 & $0.49^{\mathrm{a}}$ & $5.12^{\mathrm{b}}$ & + & {$[30,45]$} \\
\hline Taraxacum officinale Weber & 1.03 & 2.10 & 0.00 & 9.26 & +++ & {$[37]$} \\
\hline Total PSM-rich species & 42.65 & 46.93 & 24.96 & 40.79 & & \\
\hline \multicolumn{7}{|l|}{$\mathrm{T}$} \\
\hline Trifolium alpinum $\mathrm{L}$. & 24.92 & 22.89 & 10.14 & 11.61 & + & {$[38]$} \\
\hline Nardus stricta $\mathrm{L}$. & 17.40 & 22.47 & $5.58^{\mathrm{a}}$ & $22.61^{\mathrm{b}}$ & - & [37] \\
\hline Carex sempervirens Vill. & $6.41^{\mathrm{a}}$ & $20.93^{\mathrm{b}}$ & $5.42^{\mathrm{a}}$ & $18.23^{\mathrm{b}}$ & - & [37] \\
\hline Avenella flexuosa (L.) Parl. & 7.94 & 4.67 & 9.60 & 15.24 & - & [37] \\
\hline Festuca nigrescens Lam. non Gaudin & $10.95^{\mathrm{b}}$ & $0.48^{\mathrm{a}}$ & 10.76 & - & - & {$[22,37]$} \\
\hline Poa alpina $\mathrm{L}$. & 4.78 & 4.57 & 8.68 & 3.50 & - & [37] \\
\hline Geum montanum L. & 1.97 & 3.29 & 1.81 & 6.79 & + & [37] \\
\hline Festuca gr. ovina & 4.41 & - & 5.62 & - & - & [37] \\
\hline Agrostis rupestris All. & 1.36 & 2.91 & 9.12 & 10.13 & - & [37] \\
\hline Ranunculus pyrenaeus L. & 2.90 & 1.34 & 0.00 & 0.00 & + & [37] \\
\hline Total PSM-rich species & 29.80 & 27.51 & 11.95 & 18.40 & & \\
\hline
\end{tabular}

\subsection{Milk fatty acid composition}

Milk yield $(P=0.31)$ and milk total fat $(P=0.53)$ were not affected by the vegetation exploited (Tab. IV). On the contrary, significant differences occurred in FA composition between $\mathrm{F}$ and $\mathrm{T}$.

Paddock $F$ milk was richer in shortchain $(\mathrm{C} 4-\mathrm{C} 12 ; P<0.01)$ and medium- chain $(\mathrm{C} 14-\mathrm{C} 17 ; \quad P<0.05) \quad \mathrm{FAs}$, and poorer in long-chain FAs (C18-C20; $P<0.01)$. FA concentration differed by period: short- and long-chain FAs were higher during Period $1(P<0.01)$, while medium-chain FAs were higher during Period $2(P<0.001)$.

Paddock $F$ total monounsaturated FA (MUFA) content was significantly lower than 
Table II. Mean values for forage production and quality depending on pasture type (Festuca nigrescens (F) and Trifolium alpinum (T) pastures), and on the experimental period (P1 and P2). Dry matter (DM) as $\mathrm{g} \cdot 100 \mathrm{~g}^{-1} \mathrm{w} / \mathrm{w}$; ash (ASH), crude protein (CP), neutral detergent fibre (NDF), acid detergent fibre (ADF), and acid detergent lignin (ADL) as $\mathrm{g} \cdot 100 \mathrm{~g}^{-1} \mathrm{DM}$. OMD reported in percentage. Energetic value expressed as UFL $\mathrm{kg}^{-1} \mathrm{DM}$.

\begin{tabular}{|c|c|c|c|c|c|c|c|}
\hline & \multicolumn{2}{|c|}{$\mathrm{F}$} & \multicolumn{2}{|c|}{$\mathrm{T}$} & \multirow[t]{2}{*}{ SEM $^{1}$} & \multirow[t]{2}{*}{ Pasture $^{2}$} & \multirow[t]{2}{*}{ Period $^{2}$} \\
\hline & P1 & P2 & P1 & P2 & & & \\
\hline Forage production $\left(\mathrm{t} \mathrm{DM} \cdot \mathrm{ha}^{-1}\right)$ & 2.66 & 3.45 & 1.55 & 1.35 & 0.13 & $* * *$ & NS \\
\hline $\mathrm{DM}$ & 24.72 & 32.77 & 34.88 & 41.40 & 1.86 & $* * *$ & $* * *$ \\
\hline ASH & 7.12 & 7.03 & 4.05 & 4.01 & 0.05 & $* * *$ & NS \\
\hline $\mathrm{CP}$ & 12.78 & 9.88 & 14.00 & 12.84 & 0.27 & $* * *$ & $* * *$ \\
\hline NDF & 50.46 & 48.42 & 55.43 & 56.35 & 0.32 & $* * *$ & NS \\
\hline $\mathrm{ADF}$ & 28.77 & 31.16 & 31.06 & 30.13 & 0.24 & NS & NS \\
\hline ADL & 3.50 & 5.76 & 4.56 & 4.68 & 0.10 & NS & $* * *$ \\
\hline OMD & 63.03 & 58.52 & 58.75 & 56.07 & 1.70 & NS & NS \\
\hline Energetic value & 0.85 & 0.80 & 0.83 & 0.82 & 0.11 & NS & NS \\
\hline
\end{tabular}

${ }^{1}$ Standard error of the mean.

2 Statistical significance: NS: $P>0.05 ;{ }^{*} P \leq 0.05 ; * * P \leq 0.01 ; * * * P \leq 0.001$.

Table III. Daily forage intake $\left(\mathrm{kg} \cdot \mathrm{LU}^{-1} \cdot \mathrm{d}^{-1}\right)$, total fat in forage $\left(\mathrm{g} \cdot 100 \mathrm{~g}^{-1} \mathrm{DM}\right)$, and fatty acids intake $\left(\mathrm{g} \cdot \mathrm{LU}^{-1} \cdot \mathrm{d}^{-1}\right)$ by grazing animals depending on the exploited pasture type (Festuca nigrescens (F) and Trifolium alpinum (T) pastures), and on the experimental period (P1 and P2). Vegetation samples collected on $\mathrm{d} 6$ for $\mathrm{P} 1$, and on $\mathrm{d} 18$ for $\mathrm{P} 2$, the day before exploitation started into experimental paddocks.

\begin{tabular}{|c|c|c|c|c|c|c|c|}
\hline & \multicolumn{2}{|c|}{$\mathrm{F}$} & \multicolumn{2}{|c|}{$\mathrm{T}$} & \multirow[t]{2}{*}{$\mathrm{SEM}^{1}$} & \multirow[t]{2}{*}{ Pasture $^{2}$} & \multirow[t]{2}{*}{ Period $^{2}$} \\
\hline & $\mathrm{P} 1$ & $\mathrm{P} 2$ & $\mathrm{P} 1$ & $\mathrm{P} 2$ & & & \\
\hline Forage intake (DM) & 14.02 & 18.99 & 10.16 & 13.15 & 2.98 & NS & NS \\
\hline Forage fat (DM) & 1.61 & 1.49 & 1.97 & 1.85 & 0.14 & $*$ & NS \\
\hline \multicolumn{8}{|l|}{ FA intake } \\
\hline $12: 0$ & 8.66 & 8.84 & 1.76 & 1.92 & 1.24 & $* *$ & NS \\
\hline 13:0 & 1.15 & 1.17 & 1.84 & 2.00 & 0.20 & NS & NS \\
\hline 14:0 & 36.55 & 37.35 & 19.82 & 21.61 & 3.00 & $* *$ & NS \\
\hline $15: 0$ & 0.83 & 0.85 & 15.12 & 16.49 & 2.32 & $* * *$ & NS \\
\hline 16:0 & 48.49 & 49.55 & 72.78 & 79.36 & 4.71 & $* * *$ & NS \\
\hline $16: 1$ & 0.32 & 0.32 & 1.17 & 1.28 & 0.17 & $* *$ & NS \\
\hline $17: 0$ & 0.68 & 0.70 & 5.07 & 5.53 & 0.73 & $* * *$ & NS \\
\hline $17: 1$ & 5.66 & 5.79 & 8.15 & 8.89 & 2.23 & NS & NS \\
\hline 18:0 & 19.31 & 19.73 & 20.85 & 22.73 & 0.60 & NS & NS \\
\hline $18: 1 \mathrm{n}-9$ & 15.39 & 15.73 & 10.72 & 11.69 & 0.86 & $* *$ & NS \\
\hline $18: 2 \mathrm{n}-6$ & 43.45 & 44.40 & 17.37 & 18.94 & 5.93 & $*$ & NS \\
\hline $18: 3 n-6$ & 25.40 & 25.95 & 8.31 & 9.07 & 2.70 & $* * *$ & NS \\
\hline $18: 3 n-3$ & 18.77 & 19.18 & 17.04 & 18.58 & 1.57 & NS & NS \\
\hline
\end{tabular}

${ }^{1}$ Standard error of the mean.

2 Statistical significance: NS: $P>0.05 ;{ }^{*} P \leq 0.05 ; * * P \leq 0.01 ; * * * P \leq 0.001$. 
Table IV. Mean values and standard error of the mean (SEM) of daily milk yield $\left(\mathrm{kg} \cdot \mathrm{cow}^{-1} \cdot \mathrm{d}^{-1}\right)$, total fat $\left(\mathrm{g} \cdot 100 \mathrm{~g}^{-1} \mathrm{w} / \mathrm{w}\right)$, and fatty acid concentrations $\left(\mathrm{g} \cdot 100 \mathrm{~g}^{-1}\right.$ FAME) in Alpine milk from cows grazing Festuca nigrescens (F) and Trifolium alpinum (T) pastures, and for experimental period (P1 and P2). $(n=12$.

\begin{tabular}{|c|c|c|c|c|c|c|c|}
\hline & $\mathrm{F}$ & $\mathrm{T}$ & $\mathrm{P} 1$ & $\mathrm{P} 2$ & $\mathrm{SEM}^{1}$ & Pasture $^{2}$ & Period $^{2}$ \\
\hline Total fat & 4.24 & 4.00 & 4.32 & 3.92 & 0.04 & NS & NS \\
\hline C4:0 & 2.81 & 2.77 & 2.83 & 2.75 & 0.04 & NS & NS \\
\hline C6:0 & 1.77 & 1.59 & 1.73 & 1.63 & 0.03 & $* *$ & + \\
\hline C8:0 & 1.04 & 0.91 & 1.03 & 0.91 & 0.06 & $* * *$ & $* *$ \\
\hline C12:0 & 2.59 & 2.26 & 2.54 & 2.31 & 0.01 & $* * *$ & $* * *$ \\
\hline C13:0 & 0.08 & 0.11 & 0.09 & 0.09 & 0.18 & + & NS \\
\hline C14:0 & 10.17 & 9.33 & 9.44 & 10.06 & 0.05 & $* * *$ & $* * *$ \\
\hline C14:1 & 1.72 & 1.53 & 1.50 & 1.74 & 0.11 & $*$ & $* *$ \\
\hline C15:0 & 1.31 & 1.78 & 1.31 & 1.77 & 0.40 & $* *$ & $* *$ \\
\hline C17:1 & 0.29 & 0.41 & 0.36 & 0.33 & 0.10 & $* * *$ & NS \\
\hline C18:0 & 10.82 & 11.21 & 11.07 & 10.96 & 0.04 & + & NS \\
\hline C18:1 n-9 & 24.34 & 26.89 & 26.73 & 24.49 & 0.00 & $* * *$ & $* * *$ \\
\hline$t 11-\mathrm{C} 18: 1$ & 3.66 & 3.54 & 3.57 & 3.63 & 0.01 & NS & NS \\
\hline$\Sigma t$-C18:1 without $t 11-\mathrm{C} 18: 1$ & 0.31 & 0.30 & 0.29 & 0.33 & 0.52 & NS & $*$ \\
\hline C18:2 n-6 & 2.82 & 2.79 & 2.83 & 2.78 & 0.06 & NS & NS \\
\hline$c 9 t 11-\mathrm{CLA}$ & 1.59 & 1.59 & 1.54 & 1.64 & 0.04 & NS & NS \\
\hline C18:3 n-6 & 0.25 & 0.26 & 0.24 & 0.27 & 0.01 & NS & NS \\
\hline C18:3 n-3 & 1.46 & 1.02 & 1.24 & 1.24 & 0.07 & $* * *$ & NS \\
\hline C20:1 & 0.19 & 0.22 & 0.18 & 0.23 & 0.01 & + & $*$ \\
\hline$\Sigma$ short chain FA & 10.32 & 9.33 & 10.22 & 9.43 & 0.53 & $* *$ & $* *$ \\
\hline$\Sigma$ medium chain FA & 43.87 & 42.42 & 41.71 & 44.58 & 0.54 & $*$ & $* * *$ \\
\hline$\Sigma$ long chain FA & 45.73 & 48.14 & 47.98 & 45.89 & 0.50 & $* *$ & $* *$ \\
\hline$\Sigma \mathrm{n}-3$ & 1.62 & 1.14 & 1.37 & 1.39 & 0.07 & $* * *$ & NS \\
\hline$\sum \mathrm{n}-6$ & 3.20 & 3.24 & 3.22 & 3.22 & 0.06 & NS & NS \\
\hline$\Sigma \mathrm{n}-6 / \Sigma \mathrm{n}-3$ & 1.99 & 2.87 & 2.41 & 2.45 & 0.15 & $* * *$ & NS \\
\hline C14:1/C14:0 & 0.17 & 0.16 & 0.16 & 0.17 & 0.21 & NS & $*$ \\
\hline
\end{tabular}

${ }^{1}$ Standard error of the mean.

2 Statistical significance: NS: $P>0.1 ;+: P \leq 0.10 ; * P \leq 0.05 ; * * P \leq 0.01 ; * * * P \leq 0.001$.

${ }^{3}$ C4:0, C6:0, C8:0, C10:0, C12:0, C13:0, C14:0, C15:0, C16:0, C17:0, C18:0.

${ }^{4} \mathrm{C} 14: 1, \mathrm{C} 16: 1, \mathrm{C} 17: 1, \mathrm{C} 18: 1 \mathrm{n}-9, \Sigma$ t-C18:1, C20:1.

${ }_{6}^{5}$ C18:2 n-6, c9t11-CLA, C18:3 n-6, C18:3 n-3, C20:3 n-6, C20:3 n-3.

${ }^{6} \mathrm{C} 14: 1, \mathrm{C} 16: 1, \mathrm{C} 17: 1, \mathrm{C} 18: 1 \mathrm{n}-9, \Sigma \mathrm{t}$-C18:1, C18:2 n-6, c9t11-CLA, C18:3 n-6, C18:3 n-3, C20:1, C20:3 n-6, C20:3 n-3.

${ }^{7} \mathrm{C} 4: 0, \mathrm{C} 6: 0, \mathrm{C} 8: 0, \mathrm{C} 10: 0, \mathrm{C} 12: 0$.

${ }^{8}$ C13:0, C14:0, C14:1, C15:0, C16:0, C16:1, C17:0, C17:1.

${ }^{9}$ C18:0, C18:1 n-9, $\Sigma t$-C18:1, C18:2 n-6, c9t11-CLA, C18:3 n-6, C18:3 n-3, C20:1, C20:3 n-6, C20:3 n-3.

${ }^{10} \mathrm{C} 18: 3 \mathrm{n}-3, \mathrm{C} 20: 3 \mathrm{n}-3$.

${ }^{11}$ C18:2 n-6, C18:3 n-6, C20:3 n-6. 
Table V. Mean values and standard error of the mean (SEM) of total fat ( $\% \mathrm{w} / \mathrm{w}$ and $\% \mathrm{DM})$, and fatty acid concentrations ( $\mathrm{g} \cdot 100 \mathrm{~g}^{-1}$ FAME) in Nostrale d'Alpe traditional cheeses made manufacturing milk from cows grazing Festuca nigrescens $(\mathrm{F})$ and Trifolium alpinum $(\mathrm{T})$ pastures, and during experimental period (P1 and $\mathrm{P} 2)$. $(n=12$.

\begin{tabular}{|c|c|c|c|c|c|c|c|}
\hline & $\mathrm{F}$ & $\mathrm{T}$ & P1 & $\mathrm{P} 2$ & SEM $^{1}$ & Pasture $^{2}$ & Period $^{2}$ \\
\hline Total fat $(\% \mathrm{w} / \mathrm{w})$ & 28.91 & 30.82 & 30.60 & 29.13 & 0.59 & + & NS \\
\hline Total fat (\% DM) & 45.17 & 47.42 & 47.45 & 45.14 & 0.87 & NS & NS \\
\hline $\mathrm{C} 4: 0$ & 2.69 & 2.64 & 2.60 & 2.74 & 0.03 & NS & $*$ \\
\hline C6:0 & 1.70 & 1.53 & 1.58 & 1.64 & 0.03 & $* *$ & NS \\
\hline $\mathrm{C} 8: 0$ & 1.02 & 0.92 & 0.94 & 1.00 & 0.03 & $*$ & NS \\
\hline C10:0 & 2.10 & 1.78 & 1.92 & 1.96 & 0.06 & $* *$ & NS \\
\hline C12:0 & 2.59 & 2.24 & 2.38 & 2.46 & 0.07 & $* *$ & NS \\
\hline C14:0 & 10.27 & 9.31 & 9.87 & 9.71 & 0.20 & $*$ & NS \\
\hline C14:1 & 1.75 & 1.55 & 1.68 & 1.62 & 0.05 & $*$ & NS \\
\hline C15:0 & 1.36 & 1.77 & 1.78 & 1.35 & 0.12 & + & $*$ \\
\hline C16:0 & 27.83 & 26.28 & 26.52 & 27.59 & 0.43 & + & NS \\
\hline C16:1 & 1.75 & 1.80 & 1.70 & 1.85 & 0.04 & NS & NS \\
\hline $\mathrm{C} 17: 0$ & 0.78 & 1.13 & 1.02 & 0.90 & 0.06 & $* * *$ & + \\
\hline $\mathrm{C} 17: 1$ & 0.31 & 0.40 & 0.36 & 0.35 & 0.01 & $* * *$ & NS \\
\hline C18:0 & 10.86 & 11.27 & 11.20 & 10.92 & 0.10 & $*$ & NS \\
\hline C18:1 n-9 & 24.26 & 27.37 & 25.68 & 25.94 & 0.63 & $* *$ & NS \\
\hline$t 11-\mathrm{C} 18: 1$ & 3.80 & 3.66 & 4.03 & 3.43 & 0.10 & $*$ & $* * *$ \\
\hline$\Sigma t$-C18:1 without $t 11-\mathrm{C} 18: 1$ & 0.26 & 0.27 & 0.28 & 0.24 & 0.01 & NS & + \\
\hline C18:2 n-6 & 2.68 & 2.53 & 2.63 & 2.58 & 0.04 & + & NS \\
\hline$c 9 t 11-\mathrm{CLA}$ & 1.67 & 1.61 & 1.79 & 1.49 & 0.05 & NS & $* * *$ \\
\hline C18:3 n-6 & 0.26 & 0.29 & 0.26 & 0.29 & 0.01 & + & + \\
\hline C18:3 n-3 & 1.43 & 1.00 & 1.14 & 1.29 & 0.07 & $* * *$ & $*$ \\
\hline $\mathrm{C} 20: 1$ & 0.18 & 0.23 & 0.22 & 0.20 & 0.01 & NS & NS \\
\hline$\Sigma \mathrm{SFA}^{3}$ & 61.32 & 58.98 & 59.92 & 60.38 & 0.55 & $*$ & NS \\
\hline$\Sigma$ MUFA $^{4}$ & 32.30 & 35.28 & 33.95 & 33.63 & 0.61 & $*$ & NS \\
\hline$\Sigma$ PUFA $^{5}$ & 6.38 & 5.74 & 6.13 & 6.00 & 0.11 & $* * *$ & NS \\
\hline$\Sigma \mathrm{UFA}^{6}$ & 38.68 & 41.02 & 40.08 & 39.62 & 0.55 & $*$ & NS \\
\hline$\Sigma$ short chain $\mathrm{FA}^{7}$ & 10.11 & 9.12 & 9.43 & 9.80 & 0.22 & $*$ & NS \\
\hline$\Sigma$ medium chain $\mathrm{FA}^{8}$ & 44.16 & 42.34 & 43.03 & 43.47 & 0.63 & NS & NS \\
\hline$\Sigma$ long chain $\mathrm{FA}^{9}$ & 45.73 & 48.54 & 47.54 & 46.73 & 0.64 & $*$ & NS \\
\hline$\Sigma n-3^{10}$ & 1.57 & 1.11 & 1.25 & 1.43 & 0.08 & $* * *$ & $* *$ \\
\hline$\Sigma \mathrm{n}-6^{11}$ & 3.14 & 3.02 & 3.08 & 3.08 & 0.04 & NS & NS \\
\hline$\sum n-6 / \sum n-3$ & 2.01 & 2.74 & 2.54 & 2.21 & 0.12 & $* * *$ & NS \\
\hline C14:1/C14:0 & 0.17 & 0.17 & 0.17 & 0.17 & 0.00 & NS & NS \\
\hline
\end{tabular}

\footnotetext{
${ }^{1}$ Standard error of the mean.

${ }^{2}$ Statistical significance: NS: $P>0.1 ;+: P \leq 0.10 ; * P \leq 0.05 ; * * P \leq 0.01 ; * * * P \leq 0.001$.

${ }^{3}$ C4:0, C6:0, C8:0, C10:0, C12:0, C13:0, C14:0, C15:0, C16:0, C17:0, C18:0.

${ }^{4} \mathrm{C} 14: 1, \mathrm{C} 16: 1, \mathrm{C} 17: 1, \mathrm{C} 18: 1 \mathrm{n}-9, \mathrm{~s} t$-C $18: 1, \mathrm{C} 20: 1$.

${ }^{5}$ C18:2 n-6, c9t11-CLA, C18:3 n-6, C18:3 n-3, C20:3 n-6, C20:3 n-3.

${ }^{6}$ C14:1, C16:1, C17:1, C18:1 n-9, $\Sigma$ t-C18:1, C18:2 n-6, c9t11-CLA, C18:3 n-6, C18:3 n-3, C20:1, $\mathrm{C} 20: 3 \mathrm{n}-6, \mathrm{C} 20: 3 \mathrm{n}-3$.

${ }^{7} \mathrm{C} 4: 0, \mathrm{C} 6: 0, \mathrm{C} 8: 0, \mathrm{C} 10: 0, \mathrm{C} 12: 0$.

${ }^{8}$ C13:0, C14:0, C14:1, C15:0, C16:0, C16:1, C17:0, C17:1.

${ }^{9}$ C18:0, C18:1 n-9, $\Sigma t$-C18:1, C18:2 n-6, c9t11-CLA, C18:3 n-6, C18:3 n-3, C20:1, C20:3 n-6, C20:3 n-3.

${ }^{10} \mathrm{C} 18: 3 \mathrm{n}-3, \mathrm{C} 20: 3 \mathrm{n}-3$.

${ }^{11} \mathrm{C} 18: 2$ n-6, C18:3 n-6, C20:3 n-6.
} 
that found in $\mathrm{T}(P<0.001)$, related primarily to higher oleic acid concentrations. Milk from $\mathrm{F}$ contained more SFAs $(P<0.001)$ and PUFAs $(P<0.05)$ due to its higher ALA level $(1.5 \%$ vs. $1.0 \% ; P<0.001)$, even though ALA intake did not differ between T and F. SFAs were higher during Period 2 $(P<0.001)$, while MUFAs were more abundant in Period $1(P<0.001)$.

Paddock T milk contained higher concentrations of odd-linear saturated chain FAs; in particular, pentadecanoic (C15:0; $P<0.01)$ and heptadecanoic acids (C17:0; $P<0.001)$. Both these fatty acids were influenced by period (higher during Period 2; $P<0.01)$. Palmitoleic acid (C17:1; $P<0.001$ ), which was also more abundant in $\mathrm{T}$ milk, showed no period effect $(P=0.11)$.

Among C18 FAs, F milk tended to be poorer in stearic acid $(P=0.07)$, the final product of complete dietary LA and ALA reduction, as well as oleic acid ( $c 9-\mathrm{C} 18: 1$; $P<0.001)$ despite a similar intake in both vegetation types of stearic acid and higher intake in $\mathrm{F}$ of oleic acid. LA differences were never shown to be significant for either vegetation or period. The concentration of rumenic acid ( $c 9 t 11-$ CLA), the main isomer of CLAs, was similar in both $\mathrm{F}$ and $\mathrm{T}$ milk $(P=0.95)$, with no period variations $(P=0.28)$. The concentration of vaccenic acid (t11-C18:1), the main intermediate product of ruminal bio-hydrogenation of dietary LA and ALA [9], did not change with respect to vegetation $(P=0.52)$ or period $(P=0.75)$.

Vegetation did not affect $(P=0.40)$ the activity of $\Delta$ 9-desaturase, the enzyme synthesizing rumenic and oleic acid from rumen-escaped vaccenic and stearic acids, as estimated by the $\mathrm{C} 14: 1 / \mathrm{C} 14$ ratio [36].

Finally, paddock $\mathrm{F}$ milk had a higher content of eicosatrienoic acid (C20:3 n-3; $P=0.09$ ), while $\mathrm{T}$ milk was richer in dihomo- $\gamma$-linolenic acid (C20:3 n-6; $P=0.07)$.

\subsection{Cheese fatty acid composition}

Cheese and milk FA compositions were very similar and likewise affected by treatment (Tab. V). In addition, cheese produced using F milk tended to be lower in total fat $(P=0.097)$ and to contain more vaccenic acid $(P<0.05)$. Linoleic acid $(P=0.087)$ and GLA $(P=0.062)$ showed trends not usually seen in milk, with LA higher and GLA lower in paddock $F$ cheese. Compared to milk, the period seemed to affect a lower proportion of FA compounds; only rumenic and vaccenic acid contents were higher during Period $1(P<0.001)$.

\section{DISCUSSION}

Though OMD and UFL values provided evidence that the forage allowance was of a good quality, herbage chemical composition was clearly shown to be affected by the climatic conditions that occurred during summer 2007. A cold period in June, during which the average daily temperature $\left(8.2^{\circ} \mathrm{C}\right)$ was about $2{ }^{\circ} \mathrm{C}$ below time series data, combined with a drought period in July, when rainfall was almost null compared to a $90 \mathrm{~mm}$ monthly average, caused plants to age early (expressed by high DM and NDF values measured prior to the start of the experiment). During the experimental trial, forage NDF content (Tab. II) and fatty acid content (data not reported) did not vary from Period 1 to Period 2. Such pre-experimental conditions were the likely cause of the low PUFA content measured in forage compared to reference data [15]. This could have caused the resulting milk and cheese FA profiles not to be as rich in dietary PUFA compounds as expected for alpine dairy products.

We did not observe milk yield or total milk fat differences as a function of vegetation or period, although the experiment was carried out over 24 days. This result, probably related to the late mean lactation stage 
of the cows and grazing experience, gives an indication that the animals had a good energy status during the experimental course; that is, that the quality and quantity of herbage needed for cow grazing were consistently met. On the contrary, milk and cheese FA compositions, showing very similar results as expected, were influenced by the type of vegetation and the period of exploitation due to the evolution of plant phenological stage and chemical composition over time, as expressed by DM, CP, and ADL content (Tab. II), and by literature data on PSM [22, 23, 38]. Changes in milk FA composition were likely not only to be affected by animal lactation stage [36, 40], but were also affected by changes in forage chemical composition [20].

We noted a higher content of several saturated short- and medium-chain fatty acids in F milk as compared to T milk and cheese. Such differences might be related to an improved energy status (with no milk yield effects) when cows grazed $\mathrm{F}$ vegetation. In fact, reducing a negative energy balance might enhance $\mathrm{C} 4: 0-\mathrm{C} 14: 0$ and $\mathrm{C} 16: 0$ fatty acid production [43], which are synthesized mostly in the mammary gland [7].

Several factors might have improved cow energy status: (i) higher intake during $\mathrm{F}$ vegetation exploitation due to a taller sward [14] with lower NDF and DM [12] (Tab. II); (ii) higher $\mathrm{F}$ vegetation energy levels due to intense grazing and nitrogen supply; or (iii) reduced energy losses for locomotion in $\mathrm{F}$ paddocks. The first and second hypotheses failed to be supported by the experimental evidence; as no differences occurred in forage energy (Tab. II) or in DM intake although a trend was detected but unconfirmed by statistical analysis (Tab. III). Data collection to support the third hypothesis was outside the scope of this experiment, but the greater canopy height of the F sward could contribute to the reduction of cow locomotion to satisfy their feeding needs [14]. Interaction of the aforementioned factors was probably the cause of the differences observed for saturated shortand medium-chain fatty acid content. Some CLA isomers and other PUFA ruminal biohydrogenation intermediates, not quantified in this trial, might have inhibited short-chain FA de novo synthesis [43]. Consequently, we were unable to make final conclusions before fully characterizing CLA isomers in milk and derived cheese.

Paddock $F$ milk and cheese contained higher levels of total SFA, at concentrations similar to those previously recorded in other alpine production systems [9], but less than those reported for lowland milk [35].

Since medium-chain saturated fatty acids, particularly $\mathrm{C} 12: 0, \mathrm{C} 14: 0$, and C16:0, increase human plasma cholesterol concentrations [49], paddock $\mathrm{T}$ milk and cheese could be considered healthier than that derived from $\mathrm{F}$.

Paddock $\mathrm{T}$ milk and cheese were also richer in odd-linear chain FAs (C15:0 and C17:0), which are characteristic acids of ruminant milk fat. They are largely synthesized by rumen microflora, but about $10 \%$ originate from diet [48] and animal de novo synthesis from propionate [3]. The ingestion of a remarkably higher quantity of these acids in T paddocks, due to their higher concentrations in T forage (Tab. III), resulted in higher concentrations in derived milk and cheese. This fact supported the hypothesis that a direct relationship exists between the concentration of these acids in forage and their concentration levels in milk and cheese beyond that of their typical ruminal synthesis. Moreover, $\mathrm{T}$ forage was protein-rich relative to F (Tab. II), which might influence odd-linear chain acid content in milk [44].

Heptadecanoic acid content influenced the content of heptadecenoic acid (C17:1), which originates from $\Delta 9$-desaturase of heptadecanoic acid in the mammary gland [21]. Consequently, the concentration of C17:1 in $\mathrm{T}$ paddock was higher than those found in $\mathrm{F}$.

The fact that milk and derived cheese produced by cattle grazing in alpine environments are rich in beneficial functional 
fatty acids is well known [19, 34]. Of these, of particular note is n-3 PUFA, which originates from ALA and its derivatives. In our experiment, even though dairy cattle grazed in similar conditions, milk ALA concentrations varied with the precise vegetation consumed. Paddock F vs. T milk and cheese were ALA-rich, despite a lack of difference in ALA forage content and in ALA intake.

Leiber et al. [34] assumed that milk ALA could not be ingestion-related for several potential reasons: (i) body fat mobilization varies with high altitude energy deficiency; (ii) energy deficiency reduces ruminal bio-hydrogenation; or (iii) specific PSMs depress ruminal bio-hydrogenation. Under our trial conditions, the first and second reasons were not supported by the experimental evidence. In fact, while cows grazed at similar altitudes, no significant energy balance differences were estimated from herbage intake or milk yield. Only a trend for higher DM intake was recorded in F paddocks. Consequently, the principal source of ALA differences might relate to the PSM inhibitory effect on rumen bacteria.

PSM may be rumen microflora-toxic [24]. If these compounds affect the ability of the organisms to bio-hydrogenate dietary PUFA, more ALA would then escape from rumen and transfer to milk through the gut and blood. Generally, less than $10 \%$ of ALA escapes rumen reduction and reaches the duodenum; this suggested that ALA milk concentrations were surprisingly influenced by tiny rumen flora changes [34]. In our results, cows that exploited $\mathrm{F}$ vegetation, as opposed to $\mathrm{T}$, ingested a larger quantity of PSMs (Tab. I), which could have resulted in lower ruminal bio-hydrogenation of ALA. Likewise, variations in the apparent recovery of fatty acids from the duodenum to milk could have also contributed to differences in ALA content [12, 32, 41].

The concentration in milk of another beneficial FA, rumenic acid (c9t11-CLA), was comparable in our trial to that found in reference data for alpine pasture-derived milk $[8,9]$. While vegetation type affected milk ALA content, we found rumenic acid concentration to decrease with period regardless of treatment. This finding is consistent with physiological changes in plant phenology known to affect forage chemical composition [20,34]. In our trial, summer drought caused early ageing of herbage and was probably the basis for relatively low rumenic acid concentrations in milk and cheese during both periods, and could explain why our values were slightly lower than those expected at high altitude.

We found a linear relationship $\left(R^{2}=\right.$ $0.73, P<0.001)$ between concentrations of vaccenic and rumenic acids, which concurred with reports in the literature [30]. We did not observe the same behaviour in cheese where vaccenic acid content was slightly higher in $\mathrm{F}$ than in $\mathrm{T}$ pastures $(P=0.047)$, but conclusions about the difference should be considered carefully until further investigation is performed. However, such differences could confirm the lower biohydrogenation efficiency in the rumen of cows grazed on $\mathrm{F}$ pasture, being vaccenic acid a typical intermediate product of partially inhibited ruminal LA and ALA biohydrogenation [7].

More abundant stearic and oleic acids in paddock $\mathrm{T}$ rather than $\mathrm{F}$ might correlate simply to higher levels of bio-hydrogenation in the rumen of $\mathrm{T}$ animals (confirmed also by the differences in ALA concentration) given that dietary intake of both acids was similar. The measured $\mathrm{C} 14: 1 / \mathrm{C} 14: 0$ ratio also suggested that the $\Delta 9$-desaturase level was comparable.

\section{CONCLUSION}

Our experimental results showed that milk and cheese FA profiles derived from cows that were grazed on Festuca nigrescens pastures differed from the milk and cheese produced by cows that were grazed on Trifolium alpinum pastures. 
Several individually FA concentrations allowed the discrimination of milk and cheese between the treatments. Some FAs, such as odd-chain saturated acids (C15:0 and $\mathrm{C} 17: 0)$, and related unsaturated FA (C17:1), were more abundant in paddock $\mathrm{T}$ milk and cheese. Others, such as ALA (C18:3 n-3), were more abundant in $F$ paddock. The pastures differed in chemical and FA composition (proven for odd-chain saturated FAs), reflecting in FA intake and dietary PUFA ruminal biohydrogenation.

Despite the robustness of such results, many other aspects should be considered for future research, such as the effects due to changes in plant phenological stage, the relationship between vegetation and milk CLA isomer intermediates or long-chain $\mathrm{n}-3$ content, the influence of species-specific PSMs (i.e. essential oils and polyphenols) on PUFA bio-hydrogenation and flow from the rumen.

Acknowledgements: The Italian Ministries of Economy, University, Agriculture and Economic Development Research partially funded this research within the frame work of the "ProAlpe" research program (p.i. E. Piano CRA FLC Lodi), Publication No. 35. The Laboratory of Turin Chamber of Commerce performed the fatty acid analyses. The authors thank Giuseppe Rocchia for the farm management, B. Martin, Y. Chilliard, and A. Ferlay for their precious suggestions given in the revision of the manuscript.

\section{REFERENCES}

[1] Association of Official Analytical Chemist, Official methods of analysis, 15th edn., Washington, USA, 1990.

[2] Associazione Scientifica Produzioni Animali, Commissione Valutazione Alimenti, Zoot. Nutr. Anim. 8 (1982) 387-400.

[3] Cabrita A.R.J., Bessa R.J.B., Alves S.P., Dewhurst R.J., Fonseca A.J.M., Effects of dietary protein and starch on intake, milk production, and milk fatty acid profiles of dairy cows fed corn silage-based diets, J. Dairy Sci. 90 (2007) 1429-1439.

[4] Cavallero A., Talamucci P., Grignani C., Reyneri A., Caratterizzazione della dinamica produttiva di pascoli naturali italiani, Riv. Ital. Agron. 26 (1992) 325-343.

[5] Chase L.E., Energy production equations in USA at NY Hydhia Forage Laboratory, Production Agricultural Training School, Ithaca, USA, 1981.

[6] Chilliard Y., Ferlay A., Doreau M., Effect of different types of forages, animal fat or marine oils in cow's diet on milk fat secretion and composition, especially conjugated linoleic acid (CLA) and polyunsaturated fatty acids, Livest. Prod. Sci. 70 (2001) 31-48.

[7] Chilliard Y., Glasser F., Ferlay A., Bernard L., Rouel J., Doreau M., Diet, rumen biohydrogenation and nutritional quality of cow and goat milk fat, Eur. J. Lipid Sci. Technol. 109 (2007) 828-855.

[8] Collomb M., Bisig W., Butikofer U., Sieber R., Bregy M., Etter L., Seasonal variation in the fatty acid composition of milk supplied to dairies in the mountain regions of Switzerland, Dairy Sci. Technol. 88 (2008) 631-647.

[9] Collomb M., Butikofer U., Sieber R., Jeangros B., Bosset J.O., Composition of fatty acids in cow's milk fat produced in the lowlands, mountains and highlands of Switzerland using high-resolution gas chromatography, Int. Dairy J. 12 (2002) 649-659.

[10] Daget P., Poissonet J., Analyse phytologique des prairies - applications agronomiques, Centre National de la Recherche Scientifique, Montpellier, France, 1969.

[11] Dewhurst R.J., Davies D.W.R., Fisher W.J., Effects of forage NDF content and body condition score on forage intake by Holstein-Friesian dairy cows in the dry period, Animal 4 (2010) 76-80.

[12] Dewhurst R.J., Shingfield K.J., Lee M.R.F., Scollan N.D., Increasing the concentrations of beneficial polynsaturated fatty acids in milk produced by dairy cows in high-forage systems, Anim. Feed Sci. Technol. 131 (2006) 168-206.

[13] Diaz Uriarte R., Incorrect analysis of crossover trials in animal behaviour research, Anim. Behav. 63 (2002) 815-822.

[14] Dumont B., Préférences et sélection alimentaire au pâturage, Prod. Animal. 9 (1996) 359-366. 
[15] Elgersma A., Tamminga S., Dijkstra J., Lipids in herbage, in: Elgersma A., Dijikstra J., Tamminga S. (Eds.), Fresh Herbage for Dairy Cattle, Springer, The Netherlands, 2006, pp. 175-194.

[16] Elgersma A., Tamminga S., Ellen G., Modifying milk composition through forage - a review, Anim. Feed Sci. Technol. 131 (2006) 207-225.

[17] Engel E., Ferlay A., Cornu A., Chilliard Y., Agabriel C., Bielicki G., Martin B., Relevance of isotopic and molecular biomarkers for the authentication of milk according to production zone and type of feeding of the cow, J. Agric. Food Chem. 55 (2007) 90999108.

[18] Falchero L., Coppa M., Fossi A., Lombardi G., Ramella D., Tava A., Essential oil composition of lady's mantle (Alchemilla xanthochlora Rothm.) growing wild in Alpine pastures, Nat. Prod. Res. 23 (2009) 1367-1372.

[19] Ferlay A., Agabriel C., Sibra C., Journal C., Martin B., Chilliard Y., Tanker milk variability in fatty acids according to farm feeding and husbandry practices in a French semi-mountain area, Dairy Sci. Technol. 88 (2008) 193-215.

[20] Ferlay A., Martin B., Pradel P., Coulon J.B., Chilliard Y., Influence of grass-based diets on milk fatty acid composition and milk lipolytic system in Tarentaise and Montbéliarde cow breeds, J. Dairy Sci. 89 (2006) 4026-4041.

[21] Fievez V., Vlaeminck B., Dhanoa M.S., Dewhurst R.J., Use of Principal Component Analysis to investigate the origin of heptadecenoic and conjugated linoleic acids in milk, J. Dairy Sci. 86 (2003) 4047-4053.

[22] Figueredo A.C., Barroso J.G., Pedro L.G., Scheffer J.J.C., Factors affecting secondary metabolite production in plants: volatile and essential oils, Flavour Fragr. J. 23 (2008) 213-226.

[23] Fraisse D., Carnat A., Viala D., Pradel P., Besle J.M., Coulon J.B., Felgines C., Lamaison J.L., Polyphenolic composition of a permanent pasture: variations related to the period of harvesting, J. Sci. Food Agric. 87 (2007) 2427-2435.

[24] Hart K.J., Yáñez-Ruiz D.R., Duval S.M., McEwan N.R., Newbold C.J., Plant extracts to manipulate rumen fermentation, Anim. Feed Sci. Technol. 147 (2008) 8-35.
[25] International Organisation for Standardization (ISO), Animal and vegetable fats and oils - analysis by gas chromatography of methyl esters of fatty acids, ISO Standards 5508, 1990.

[26] International Organisation for Standardization (ISO), Animal and vegetable fats and oils - preparation of methyl ester of fatty acids, Clause 5: trans-esterification method, ISO Standards 5509, 2000.

[27] Jones D.I.H., Hayward M.V., The effect of pepsin pre-treatment of herbage on the prediction of dry matter digestibility from solubility in fungal cellulose solutions, J. Sci. Food Agric. 26 (1975) 711-718.

[28] Jouglet J.P., Dorée A., Le pâturage mixte ovin - équin. Incidence sur la dynamique d'une pelouse subalpine à Fétuque paniculée, Doc. CEMAGREF-INERM n ${ }^{\circ} 215$, St. Martin d'Hères, France, 1987.

[29] Kenward M.G., Jones B., Design and Analysis of Cross-Over Trials, 2nd edn., Chapman \& Hall/CRC Press, London, UK, 2003.

[30] Khanal R.C., Dhiman T.R., Biosynthesis of conjugated linoleic acid (CLA): a review, Pak. J. Nutr. 3 (2004) 72-81.

[31] Lebreton P., Gallet C., Les communautés végétales sont biochimiquement organisées, Acta Bot. Gallica 154 (2007) 573-595.

[32] Lee M.R.F., Tweed J.K.S., Dewhurst R.J., Scollan N.D., Effect of forage: concentrate ratio on ruminal metabolism and duodenal flow of fatty acids in beef steers, Anim. Sci. 82 (2006) 31-40.

[33] Leiber F., Kreuzer M., Jörg B., Leuenberger H., Wettstein H.R., Contribution of altitude and Alpine origin of forage to the influence of Alpine sojourn of cows on intake, nitrogen conversion, metabolic stress and milk synthesis, Anim. Sci. 78 (2004) 451-466.

[34] Leiber F., Kreuzer M., Nigg D., Wettstein H.R., Scheeder M.R.L., A study on the causes for the elevated n-3 fatty acids in cows' milk of Alpine origin, Lipids 40 (2005) 191-202.

[35] Lindmark Månsson H., Fatty acids in bovine milk fat, Food Nutr. Res. 52 (2008) Doi: 10.3402/fnr.v52i0.1821.

[36] Lock A.L., Garnsworthy P.C., Seasonal variation in milk conjugated linoleic acid and delta9-desaturase activity in dairy cows, Livest. Prod. Sci. 79 (2003) 47-59.

[37] Lourenço M., Vlaeminck B., Bruinenberg M., Demeyer D., Fievez V., Milk fatty acid composition and associated rumen lipolysis 
and fatty acid hydrogenation when feeding forages from intensively managed or seminatural grasslands, Anim. Res. 54 (2005) 471-484.

[38] Mariaca R., Berger T., Gauch R., Imhof M., Jeangros B., Bosset J.O., Occurrence of volatile mono- and sesquiterpenoids in highland and lowland plant species as possible precursors for flavor compounds in milk and dairy products, J. Agric. Food Chem. 45 (1997) 4423-4434.

[39] Oleszek W., Stochmal A., Janda B., Concentration of isoflavones and other phenolics in the aerial parts of Trifolium species, J. Agric. Food Chem. 55 (2007) 8095-8100.

[40] Palmquist D.L., Beaulieu D., Barbano D.M., Feed and animal factors influencing milk fat composition, J. Dairy Sci. 76 (1993) 1753-1771.

[41] Sackmann J.R., Duckett S.K., Gillis M.H., Realini C.E., Parks A.H., Eggelston R.B., Effects of forage and sunflower oil levels on ruminal biohydrogenation of fatty acids and conjugated linoleic acid formation in beef steers fed finishing diets, J. Anim. Sci. 81 (2003) 3174-3181.

[42] Shingfield K.J., Chilliard Y., Toivonen V., Kairenius P., Givens D.I., Trans Fatty Acids and Bioactive Lipids in Ruminant Milk, in: Bösze Z. (Ed.), Advances in Experimental Medicine and Biology - Bioactive Components of Milk, Springer, New York, USA, 2008, pp. 3-65.
[43] Shingfield K.J., Griinari J.M., Role of biohydrogenation intermediates in milk fat depression, Eur. J. Lipid Sci. Technol. 109 (2007) 799-816.

[44] Shingfield K.J., Reynolds C.K., Lupoli B., Toivonen V., Yurawecz M.P., Delmonte P., Griinari J.M., Grandison A.S., Beever D.E., Effect of forage type and proportion of concentrate in the diet on milk fatty acid composition in cows given sunflower oil and fish oil, Anim. Sci. 80 (2005) 225-238.

[45] Smit H.J., Taweel H.Z., Tas B.M., Tamminga S., Elgersma A., Comparison of techniques for estimating herbage intake of grazing dairy cows, J. Dairy Sci. 88 (2005) 1827-1836.

[46] Tava A., Ramella D., Grecchi M., Aceto P., Paoletti R., Piano E., Volatile constituents of Trifolium pratense and T. repens from N.E. Italian Alpine pastures, Nat. Prod. Comm. 4 (2009) 835-838.

[47] Thiex N.J., Anderson S., Gildemeister B., Crude Fat, Hexanes Extraction, in Feed, Cereal Grain, and Forage (Randall/Soxtec/ Submersion Method): Collaborative Study, J. AOAC Int. 86 (2003) 899-908.

[48] Vlaeminck B., Fievez V., Cabrita A.R.J., Fonseca A.J.M., Dewhurst R.J., Factors affecting odd- and branched-chain fatty acids in milk: a review, Anim. Feed Sci. Technol. 131 (2006) 389-417.

[49] Williams C.M., Dietary fatty acids and human health, Ann. Zootech. 49 (2000) 165-180. 